\title{
Clinical Trajectories, Healthcare Resource Use, and Costs of Diabetic Nephropathy Among Patients with Type 2 Diabetes: A Latent Class Analysis
}

\author{
Ruixuan Jiang · Ernest Law - Zhou Zhou - Hongbo Yang • \\ Eric Q. Wu $\cdot$ Raafat Seifeldin
}

Received: January 31, 2018 / Published online: March 29, 2018

(C) The Author(s) 2018

\section{ABSTRACT}

Introduction: Patients with type 2 diabetes mellitus (T2DM) are clinically heterogeneous in terms of disease severity, treatment, and comorbidities, potentially resulting in differential diabetic nephropathy (DN) progression courses. In this exploratory study we used latent class analysis (LCA) to identify patient groups with distinct clinical profiles of T2DM severity and explored the association between disease severity, DN progression or reversal, and healthcare resource use (HRU) and costs.

Methods: Latent class analysis was used to group adults with $\geq 2$ medical claims with a

Enhanced content To view enhanced content for this article go to https://doi.org/10.6084/m9.figshare. 5977249.

Electronic supplementary material The online version of this article (https://doi.org/10.1007/s13300018-0410-8) contains supplementary material, which is available to authorized users.

R. Jiang · E. Law $(\bowtie)$

Department of Pharmacy Systems, Outcomes, and Policy, College of Pharmacy, University of Illinois at Chicago, Chicago, IL, USA

e-mail: elaw3@uic.edu

Z. Zhou · H. Yang · E. Q. Wu

Analysis Group, Inc., Boston, MA, USA

R. Seifeldin

Formerly of Takeda Development Center Americas, Inc., Deerfield, IL, USA diagnosis of T2DM and $\geq 2$ urine albumin tests within the Truven MarketScan database (2004-2014), based on T2DM-related complications, comorbidities, and therapies. DN severity categories (normoalbuminuria, moderately increased albuminuria, and severely increased albuminuria) were determined based on urine albumin measure. The risks of $\mathrm{DN}$ progression and reversal (change to a more/less severe DN category) were compared among all identified latent classes using Kaplan-Meier analyses and log-rank tests. All-cause and DNrelated costs and HRU were assessed and compared during the study period among the identified latent classes.

Results: Four clinically distinct profiles were identified among the 23,235 eligible patients: low comorbidity/low treatment $(46.5 \%)$, low comorbidity/high treatment $(29.0 \%)$, moderate comorbidity/high insulin use $(9.7 \%)$, and high comorbidity/moderate treatment $(14.8 \%)$. The 5 -year DN progression rates for these clinically distinct profiles were $11.8,18,16.5$, and $27.7 \%$, respectively. Compared with the low comorbidity/low treatment group, all other groups were associated with an increased risk of DN progression (all $p<0.001$ ). Increasing comorbidity was significantly associated with higher all-cause and DN-related HRU and costs, primarily driven by higher pharmacy and inpatient costs.

Conclusion: Patients with T2DM who have more comorbidities experienced higher rates of 
DN progression and HRU and incurred higher healthcare costs compared with patients with low comorbidity profiles. Future prospective studies are needed to confirm the significance of these groups on DN progression, HRU, and costs.

Funding: Takeda Development Center Americas, Inc.

Keywords: Clinical outcomes; Costs; Diabetic nephropathy; Healthcare resource use; Type 2 diabetes

\section{INTRODUCTION}

Diabetic nephropathy (DN), often referred as diabetic kidney disease, is a common and serious complication of type 2 diabetes mellitus (T2DM), and presents as albuminuria and/or decreased glomerular filtration rate (GFR) [1-3]. In addition to being a leading cause of end-stage renal disease (ESRD), DN greatly increases the risk of all-cause and cardiovascular-related mortality, cardiovascular disease and coronary atherosclerosis, and kidney failure among patients with diabetes $[4,5]$. DN affects up to $40 \%$ of patients with T2DM [6] and had an estimated prevalence of $3.3 \% \quad(6.9$ million adults) within the USA during 2005-2008 [7]. The overall prevalence of DN among U.S. adults with diabetes did not change significantly from 1988 to 2014 [8]. The disease progression of DN is classified by the following stages based on urine albumin levels: normoalbuminuria $(<30 \mathrm{mg} / 24 \mathrm{~h}$ or an albumin/creatinine ratio [ACR] of $<30 \mu \mathrm{g} / \mathrm{mg}$ ), moderately increased albuminuria $(30-300 \mathrm{mg} / 24 \mathrm{~h}$ or an ACR of $30-300 \mu \mathrm{g} / \mathrm{mg}$ ), and severely increased albuminuria $(>300 \mathrm{mg} / 24 \mathrm{~h}$ or an ACR $>300 \mu \mathrm{g}$ / mg) [9]. Moderately increased albuminuria precedes severely increased albuminuria, and without treatment, the GFR progressively declines and can ultimately result in ESRD [10]. However, the severity of T2DM is clinically heterogeneous among affected patients, which may also result in differential courses of $\mathrm{DN}$ progression [11].

Several prior studies have identified risk factors for DN progression. The United Kingdom
Prospective Diabetes Study (UKPDS) examined the progression of $\mathrm{DN}$ among 5100 T2DM patients followed for over a decade [12]. The UKPDS reported the rates and time to DN progression among patients with normoalbuminuria, moderately increased albuminuria, or severely increased albuminuria, and found that the more severe the albuminuria, the shorter the time to progression to the next stage of DN [12]. In addition, in T2DM patients, long duration and poor control have been associated with DN progression [13], while the treatment of metabolic syndrome has been shown to be independently associated with lesser progression of DN in T2DM [14]. A prospective study of Japanese patients with T2DM examined the frequency of and reasons for DN reversal and found that DN duration, DN treatment type, glycated hemoglobin (HbA1c) levels, and healthy blood pressure were linked to likelihood of reversal [15]. The relationship between factors related to treatment type and comorbidity cannot be assumed to lie on a continuum, but rather may co-vary in different ways in different patients, resulting in distinct clinical patterns. These patterns, or their relationship to meaningful outcomes, have not been explored.

Understanding the relationships between DN progression and T2DM comorbidity and severity, as well as which patients are at particular risk of progression or poor outcomes, may help improve patient-centered disease management. The use of real-world patient data from claims databases to characterize T2DM could contribute to this goal, as real-world patients have been shown to be more heterogeneous in terms of both disease severity and diabetic treatments received [16], and claims data are a large and accessible resource. Latent class analysis (LCA), a statistical method for grouping individuals who share common characteristics and thereby allowing distinct "clusters" to be identified [17], can integrate the limited disease information available in claims databases. LCA has been previously used to examine heterogeneity in patient populations, to identify subgroups at high potential risk of disability or adverse effects, or to predict patients who may benefit from particular interventions in various disease areas [18-21]. 
In the study reported here, we used LCA to integrate multiple indicators of T2DM severity that are readily available in commonly used claims databases to identify patient groups with distinct clinical profiles. Differences in the clinical trajectory of DN (i.e., DN progression and reversal) were then assessed among the identified latent classes. Additionally, as there is limited information regarding the healthcare economic burden among patient groups with distinct clinical profiles of T2DM, we assessed the health resource utilization and healthcare costs among the patient groups identified with LCA and identified the patient subgroup with the highest economic burden.

\section{METHODS}

\section{Data Source}

This study used data from the Truven Health Analytics MarketScan ${ }^{\circledR}$ Commercial and Medicare Supplemental and Lab databases (1 January 2003 to 31 December 2014), which represent approximately 25 million U.S. employees, dependents, and Medicare-eligible retirees covered by over 130 health plans and self-insured employers. A subset of the covered lives (approx. 1 million) have recorded laboratory tests (mainly ordered in office-based practice) in the MarketScan ${ }^{\circledR}$ Lab Database. Data were de-identified and comply with the patient confidentiality requirements of the U.S. Health Insurance Portability and Accountability Act; thus, no institutional review was required.

\section{Study Population and Study Cohorts}

The study sample included adult patients (aged $\geq 18$ years) with $\geq 2$ distinct medical claims with a diagnosis of T2DM (International Classification of Diseases, 9th revision-Clinical Modification [ICD-9-CM]: 250.x0, 250.x2) and with $\geq 2$ urine albumin test results after the first observed T2DM diagnosis. Patients with at least one eligible urine albumin test who met the following criteria were identified: (1) with $\geq 1$ additional follow-up urine albumin test; (2) with continuous enrollment for $\geq 12$ months prior to and 6 months following the test date; and (3) without ESRD during the 12 months prior to the test date (Fig. 1). The index date was randomly selected among all eligible urine albumin test dates.

\section{Study Measures and Outcomes}

Type 2 diabetes mellitus severity indicators (diabetic comorbidities, complications, and treatments) included in the LCA were assessed during the 12-month period prior to the index date. T2DM-related complications and comorbidities included retinopathy, neuropathy, ischemic heart disease, cerebrovascular disease, chronic heart failure, hypertension, chronic kidney disease (CKD)-related symptoms (hyperkalemia, high parathyroid hormone level, and high phosphorus level), and metabolic disorder (lipid disorders and other metabolic disorders) (see Appendix A in the Electronic Supplementary Material [ESM]). Diabetic treatments included insulin, metformin, sulphonylureas, dipeptidyl peptidase-4 inhibitor, glucagon-like peptide-1-based therapy, and other antidiabetic agents (amylin analogs, amino acid derivatives, meglitinide analogs, aldose reductase inhibitors, alpha-glucosidase inhibitors, dopamine receptor agonists, insulin sensitizing agents and antidiabetic combinations) (see ESM Appendix B).

\section{Baseline Characteristics}

Baseline characteristics (demographics, disease characteristics, and DN-related treatments) were assessed during the 12-month period prior to the index date. Patient demographic information collected included age, sex, and type of health insurance. The time from the first observed T2DM diagnosis to the index date and patients' DN severity at the index date (normoalbuminuria, moderately increased albuminuria, or severely increased albuminuria) were assessed. Normoalbuminuria was defined as urinary albumin excretion of $<30 \mathrm{mg} / 24 \mathrm{~h}$ or an ACR of $<30 \mu \mathrm{g} / \mathrm{mg}$; moderately increased albuminuria was defined as excretion of $30-300 \mathrm{mg} / 24 \mathrm{~h}$ or an ACR of $30-300 \mu \mathrm{g} / \mathrm{mg}$; 
Abbreviations: ESRD = end-stage renal disease; $\mathrm{N}=$ number.

Patients with $\geq 2$ medical claims with an associated diagnosis for T2DM

$\mathrm{N}=8,065,232$

Patients with $\geq 1$ urine albumin test result after the first observed T2DM diagnosis

$\mathrm{N}=152,640$

Patients with $\geq 1$ follow-up urine albumin test result after any potential index date

$\mathrm{N}=57,275$

Patients with $\geq 12$ months of continuous enrollment in a health plan prior to any potential index date

$\mathrm{N}=\mathbf{2 4 , 9 5 4}$

Patients with continuous enrollment in a health plan for $\geq 6$ months and through the subsequent follow-up urine albumin test date following any potential index date

$\mathrm{N}=23,512$

Patients without ESRD during the 12 months prior to any potential index date

$\mathrm{N}=\mathbf{2 3 , 4 0 2}$

Patients aged $\geq 18$ years as of any potential index date

$\mathrm{N}=23,235$

Random selection of index date for each patient

$\mathrm{N}=\mathbf{2 3 , 2 3 5}$

Normoalbuminuria Cohort

$N=18,409$

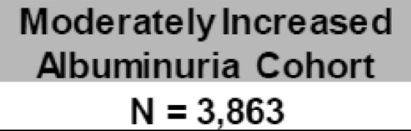

$\mathrm{N}=3,863$
Severely Increased

Abuminuria Cohort

$\mathrm{N}=963$

Fig. 1 Sample selection. ESRD End-stage renal disease, T2DM type 2 diabetes mellitus

severely increased albuminuria was defined as excretion of $>300 \mathrm{mg} / 24 \mathrm{~h}$ or an ACR $>300 \mu \mathrm{g} /$ mg. In addition, for each patient the Charlson Comorbidity Index (CCI) score [22] and the use of DN-related treatments during the 12-month period before the index date were recorded. DNrelated treatments included angiotensin converting enzyme inhibitors, angiotensin receptor blockers, diuretics, calcium channel blockers, and other antihypertensive agents [23] (see ESM Appendix B).

\section{Progression or Reversal of DN}

The time to DN progression and time to DN reversal were assessed from the index date until inpatient mortality, the end of continuous eligibility, or the end of data availability, whichever came first. DN severity was classified into four disease stages ranging from normal to most severe: normoalbuminuria, moderately increased albuminuria, severely increased albuminuria, and presence of ESRD/dialysis/renal transplantation procedure (see ESM Appendix C). DN disease progression was defined as the 
presence of a urine albumin test, diagnosis or procedure indicating a more severe disease stage than the index disease stage, while DN disease reversal was defined as the presence of a urine albumin test indicating a less severe disease stage than the index disease stage. DN disease reversal was only assessed in patients with moderately increased albuminuria and severely increased albuminuria at the index date.

\section{Healthcare Resource Use and Costs}

Economic outcomes were assessed at the perpatient-per-year (PPPY) level from the index date until 2 years from the index date, the end of continuous eligibility, end of data availability, or inpatient mortality, whichever came first. Healthcare resource use (HRU) information was collected for all-cause and DN-related medical visits, including inpatient, emergency room (ER), outpatient, and other medical visits. DNrelated HRU was defined as medical services associated with a diagnosis of DN or kidney disease, or a procedure for dialysis/hemodialysis or renal transplantation.

All-cause and nephropathy-related healthcare costs were calculated from a U.S. payer's perspective and inflated to 2016 U.S. dollars using the annual medical care component of the Consumer Price Index [24]. Cost components included medical costs (inpatient, ER, outpatient, and other medical services costs) and pharmacy costs. DN-related costs were defined as costs associated with a diagnosis code of diabetic nephropathy or costs associated with a procedure for dialysis/hemodialysis or renal transplantation.

\section{Statistical Analysis}

Latent class analysis was used to identify groups of patients with clinically distinct T2DM severity profiles. Class membership was determined based on the T2DM disease severity and treatment indicators, and individual patients could belong to only one group. Models with a varying number of classes were estimated, and the best-fitting model was chosen. Model selection was based on the consideration of several criteria, including model fit statistics (Bayesian
Information Criterion and Akaike Information Criterion) and interpretability of groups. Once the model was selected, subjects were assigned to the latent class based on their probability of being in that class. Analyses were conducted in SAS version 9.4 software using the PROC LCA procedure [25].

For baseline characteristics, means and standard deviations (SD) were reported for continuous characteristics; frequencies and percentages were reported for categorical characteristics. Characteristics were compared between the selected reference latent class versus other latent classes using Wilcoxon ranksum tests for continuous variables and Chisquare tests for categorical variables. Time to disease progression and time to disease reversal were evaluated for each latent class using Kaplan-Meier methods and compared between the reference latent class versus other classes using log-rank tests. The incidence rates of each type of healthcare visit PPPY and the annual healthcare costs were described for each latent class and compared between the reference latent class versus other classes using Wilcoxon rank-sum tests. A $p$ value of $\leq 0.05$ was considered to be statistically significant.

All procedures followed were in accordance with the ethical standards of the responsible committee on human experimentation (institutional and national) and with the Helsinki Declaration of 1964, as revised in 2013. Only de-identified data was used in this study, thus no institutional review was required. This article is based on previously conducted studies and does not contain any studies with human participants or animals performed by any of the authors.

\section{RESULTS}

\section{Latent Class Analysis}

A total of 23,235 patients with T2DM fulfilled all study criteria and were included in the analysis, including 18,409 patients with normoalbuminuria, 3863 with moderately increased albuminuria, and 963 with severely increased albuminuria (Fig. 1). In the LCA, a 
four-class model yielded the best fit, and four clinically distinct T2DM patient profiles were identified based on distributions of complications/comorbidities (e.g., microvascular and cardiovascular disease, CKD-related symptoms, and metabolic disorder) and use of diabetic treatment (e.g., insulin, metformin, etc.) (Table 1). The four clinically distinct profiles were defined as: (1) Latent Class 1 (46.5\% of the sample $[N=10,812]$; reference latent class), low comorbidity/low treatment; (2) Latent Class 2 $(29.0 \% \quad[N=6728])$, low comorbidity/high treatment; (3) Latent Class $3(9.7 \%$ [ $N=2255])$, moderate comorbidity and high insulin use; (4) Latent Class $4 \quad(14.8 \% \quad[N=3440])$, high comorbidity and moderate treatment. Retinopathy was more common among Latent Classes 3 (26.8\%) and 4 (24.4\%) compared with Latent Classes 1 (2.6\%) and $2(13.3 \%)$, and Latent Class 4 had a higher incidence of all other comorbidities in comparison with the other three latent classes.

\section{Baseline Demographics and Disease Characteristics}

The baseline demographics and disease characteristics of the four latent classes are described in Table 2; multiple significant differences existed between the characteristics of the latent classes. For example, patients with moderate comorbidity/high insulin use were younger (mean [SD] 49.1 [13.3] years) than those in the low comorbidity/low treatment group (54.5 [9.2] years), and patients with high comorbidity/moderate treatment were older (58.2 [8.7] years) and more likely to be male than those in the low comorbidity/low treatment group (male 57 vs. $54 \%$, respectively; all $p<0.05$ ). Patients from the southern USA were over-represented in every class (range 38-44\%). Preferred provider organization was the most common type of health plan among all classes (range 72-76\%).

The majority of patients in all groups were classified with normoalbuminuria at the index date (range 68-84\%); however, higher proportions of patients were classified with moderately increased albuminuria or severely increased albuminuria in other groups compared with the low comorbidity/low treatment group (all $p<0.05$ ) (Table 2). Compared with the low comorbidity/low treatment group, all other groups had significantly longer mean time since first observed T2DM diagnosis (low comorbidity/low treatment: 32.9 [SD 25.1] months; low comorbidity/high treatment: 42.0 [26.8] months; moderate comorbidity/high insulin use: 42.6 [26.9] months; high comorbidity/moderate treatment: 46.6 [29.6] months; all $p<0.01)$. Patients in the high comorbidity/moderate treatment group had the highest mean CCI (mean 2.9 [SD 1.6]), followed by the moderate comorbidity/high insulin group (1.7 [1.1]), the low comorbidity/high treatment group (1.6 [1.0]), and the low comorbidity/low treatment group (1.4 [1.0]). All other groups had significantly higher mean CCI compared with the low comorbidity/low treatment group (all $p<0.05)$.

\section{Time to DN Disease Progression}

The median years of follow-up for DN progression among the low comorbidity/low treatment, low comorbidity/high treatment, moderate comorbidity/high insulin use, and high comorbidity/moderate treatment groups were $2.1,1.9,2.1$, and 1.6 years, respectively. The respective 1-, 3-, and 5-year DN progression rates were $9.3,20.8$, and $27.7 \%$ for the high comorbidity/moderate treatment group; 5.9, 13.3 , and $16.5 \%$ for the moderate comorbidity/ high insulin group; 5.6, 13.9, and $18.0 \%$ for the low comorbidity/high treatment group; and $4.0,9.6$, and $11.8 \%$ for the low comorbidity/low treatment group (Fig. 2). Compared to the low comorbidity/low treatment group, all other groups were associated with a significantly increased risk of progression to a more severe stage of DN (all $p<0.01$ ). The high comorbidity/moderate treatment group was associated with the highest risk of disease progression among all latent groups.

\section{Time to DN Disease Reversal}

The median years of follow-up for DN reversal among the low comorbidity/low treatment, low 
Table 1 Item-response probabilities for the four-class model: probability of each patient in a given latent class

\begin{tabular}{|c|c|c|c|c|}
\hline \multirow[t]{2}{*}{ Item } & \multicolumn{4}{|l|}{ Latent class (\%) } \\
\hline & $\begin{array}{l}\text { Latent Class } 1 \text { (low } \\
\text { comorbidity/low } \\
\text { treatment group) }\end{array}$ & $\begin{array}{l}\text { Latent Class } 2 \text { (low } \\
\text { comorbidity/high } \\
\text { treatment group) }\end{array}$ & $\begin{array}{l}\text { Latent Class } 3 \\
\text { (moderate } \\
\text { comorbidity/high } \\
\text { insulin use group) }\end{array}$ & $\begin{array}{l}\text { Latent Class } 4 \text { (high } \\
\text { comorbidity/moderate } \\
\text { treatment group) }\end{array}$ \\
\hline \multicolumn{5}{|l|}{ Comorbidity } \\
\hline \multicolumn{5}{|c|}{ Microvascular disease } \\
\hline $\begin{array}{l}\text { Retinopathy } \\
\text { disease }\end{array}$ & 2.6 & 13.1 & 26.8 & 24.4 \\
\hline $\begin{array}{l}\text { Neuropathy } \\
\text { disease }\end{array}$ & 5.2 & 13.3 & 16.4 & 31.7 \\
\hline \multicolumn{5}{|c|}{ Cardiovascular disease } \\
\hline $\begin{array}{l}\text { Ischemic heart } \\
\text { disease }\end{array}$ & 6.8 & 6.2 & 4.7 & 40.7 \\
\hline $\begin{array}{l}\text { Cerebrovascular } \\
\text { disease }\end{array}$ & 2.4 & 1.3 & 2.0 & 19.0 \\
\hline $\begin{array}{l}\text { Chronic heart } \\
\text { failure }\end{array}$ & 0.6 & 0.1 & 1.6 & 11.0 \\
\hline Hypertension & 58.2 & 64.0 & 32.8 & 94.7 \\
\hline $\begin{array}{l}\text { CKD-related } \\
\text { disease }^{a}\end{array}$ & 0.5 & 0.9 & 1.0 & 5.3 \\
\hline $\begin{array}{l}\text { Metabolic } \\
\text { disorder }^{\mathrm{b}}\end{array}$ & 64.1 & 72.1 & 43.3 & 92.8 \\
\hline \multicolumn{5}{|c|}{ Use of diabetic treatment } \\
\hline Metformin & 59.8 & 88.2 & 18.3 & 56.3 \\
\hline Sulphonylureas & 13.5 & 48.3 & 3.5 & 25.9 \\
\hline Insulin & 0.0 & 21.9 & 87.7 & 44.1 \\
\hline DPP4 inhibitor & 4.6 & 19.3 & 0.7 & 12.8 \\
\hline $\begin{array}{l}\text { GLP1-based } \\
\text { therapy }\end{array}$ & 1.5 & 15.2 & 3.1 & 9.5 \\
\hline $\begin{array}{l}\text { Other antidiabetic } \\
\text { agents }^{c}\end{array}$ & 7.6 & 35.5 & 31.3 & 26.5 \\
\hline
\end{tabular}

Item-response probabilities were calculated among all patients, given the probability of each patient being in that latent class CKD Chronic kidney disease, DPP4 dipeptidyl peptidase-4, GLP-1 glucagon-like peptide-1

${ }^{a}$ CKD-related disease included hyperkalemia, high parathyroid hormone level, and high phosphorus level

${ }^{b}$ Metabolic disorder included lipid disorders and other metabolic disorders

c Other antidiabetic agents included amylin analogs, amino acid derivatives, meglitinide analogs, aldose reductase inhibitors, alpha-glucosidase inhibitors, dopamine receptor agonists, insulin sensitizing agents, and antidiabetic combinations 
Table 2 Patient baseline demographics and disease characteristics according to latent class

\begin{tabular}{|c|c|c|c|c|c|c|c|}
\hline \multirow{2}{*}{$\begin{array}{l}\text { Patient baseline demographics } \\
\text { and disease characteristics }\end{array}$} & \multirow{2}{*}{$\begin{array}{l}\text { Latent Class } \\
1 \\
(N=10,812)\end{array}$} & \multirow{2}{*}{$\begin{array}{l}\text { Latent } \\
\text { Class } 2 \\
(N=6728)\end{array}$} & \multirow{2}{*}{$\begin{array}{l}\text { Latent } \\
\text { Class } 3 \\
(N=2255)\end{array}$} & \multirow{2}{*}{$\begin{array}{l}\text { Latent } \\
\text { Class } 4 \\
(N=3440)\end{array}$} & \multicolumn{3}{|l|}{$p$ value $^{\mathrm{a}}$} \\
\hline & & & & & $\begin{array}{l}2] \text { vs. } \\
{[1]}\end{array}$ & $\begin{array}{l}{[3] \text { vs. }} \\
{[1]}\end{array}$ & $\begin{array}{l}\text { [4] vs. } \\
{[1]}\end{array}$ \\
\hline Age at index date (years) & $54.5 \pm 9.2$ & $54.4 \pm 8.6$ & $49.1 \pm 13.3$ & $58.2 \pm 8.7$ & & $<0.01$ & $<0.001$ \\
\hline Male & $5795(54 \%)$ & $3789(56 \%)$ & $1199(53 \%)$ & $1965(57 \%)$ & $<0.05$ & & $<0.001$ \\
\hline \multicolumn{8}{|l|}{ U.S. region } \\
\hline Northeast & $2016(19 \%)$ & $1196(18 \%)$ & $394(17 \%)$ & $837(24 \%)$ & & & $<0.001$ \\
\hline North-Central & $2826(26 \%)$ & $1629(24 \%)$ & $722(32 \%)$ & $828(24 \%)$ & $<0.05$ & $<0.01$ & $<0.001$ \\
\hline South & $4533(42 \%)$ & $2976(44 \%)$ & $855(38 \%)$ & $1367(40 \%)$ & $<0.05$ & $<0.01$ & $<0.001$ \\
\hline West & $1434(13 \%)$ & $927(14 \%)$ & $283(13 \%)$ & $408(12 \%)$ & & & $<0.001$ \\
\hline \multicolumn{8}{|l|}{ Insurance plan type } \\
\hline der organization & $8094(75 \%)$ & $5145(76 \%)$ & $1678(74 \%)$ & $2483(72 \%)$ & $<0.05$ & & $<0.001$ \\
\hline Non-capitated point-of-service & $781(7 \%)$ & $538(8 \%)$ & $166(7 \%)$ & $225(7 \%)$ & & & \\
\hline Exclusive provider & $501(5 \%)$ & $343(5 \%)$ & $82(4 \%)$ & $228(7 \%)$ & & $<0.01$ & $<0.001$ \\
\hline Comprehensive & $1025(9 \%)$ & $502(7 \%)$ & $198(9 \%)$ & $442(13 \%)$ & $<0.05$ & & $<0.001$ \\
\hline Consumer-driven health plan & $314(3 \%)$ & $141(2 \%)$ & $99(4 \%)$ & $43(1 \%)$ & $<0.05$ & $<0.01$ & $<0.001$ \\
\hline High-deductible health plan & $97(1 \%)$ & $59(1 \%)$ & $32(1 \%)$ & $19(1 \%)$ & & $<0.01$ & $<0.001$ \\
\hline $\begin{array}{l}\text { Time from first observed T2DM } \\
\text { diagnosis in the database to the } \\
\text { index date (months) }\end{array}$ & $32.9 \pm 25.1$ & $42.0 \pm 26.8$ & $42.6 \pm 26.9$ & $46.6 \pm 29.6$ & $<0.05$ & $<0.01$ & $<0.001$ \\
\hline \multicolumn{8}{|c|}{ Diabetic nephropathy disease status at the index date } \\
\hline Normal & $9083(84 \%)$ & $5249(78 \%)$ & $1728(77 \%)$ & $2349(68 \%)$ & $<0.05$ & $<0.01$ & $<0.001$ \\
\hline $\begin{array}{l}\text { Moderately increased } \\
\text { albuminuria }\end{array}$ & $1483(14 \%)$ & $1208(18 \%)$ & $400(18 \%)$ & $772(22 \%)$ & $<0.05$ & $<0.01$ & $<0.001$ \\
\hline Severely increased albuminuria & $246(2 \%)$ & $271(4 \%)$ & $127(6 \%)$ & $319(9 \%)$ & $<0.05$ & $<0.01$ & $<0.001$ \\
\hline Charlson Comorbidity Index & $1.4 \pm 1.0$ & $1.6 \pm 1.0$ & $1.7 \pm 1.1$ & $2.9 \pm 1.6$ & $<0.05$ & $<0.01$ & $<0.001$ \\
\hline Nephropathy-related treatments & $6892(64 \%)$ & $5369(80 \%)$ & $1352(60 \%)$ & $2975(86 \%)$ & $<0.05$ & $<0.01$ & $<0.001$ \\
\hline ACE inhibitor & $3045(28 \%)$ & $2823(42 \%)$ & $782(35 \%)$ & $1524(44 \%)$ & $<0.05$ & $<0.01$ & $<0.001$ \\
\hline Diuretic & $1646(15 \%)$ & $1291(19 \%)$ & $327(15 \%)$ & $1201(35 \%)$ & $<0.05$ & & $<0.001$ \\
\hline Calcium channel blocker & $1339(12 \%)$ & $1017(15 \%)$ & $225(10 \%)$ & $856(25 \%)$ & $<0.05$ & $<0.01$ & $<0.001$ \\
\hline $\mathrm{ARB}$ & $1208(11 \%)$ & $981(15 \%)$ & $262(12 \%)$ & $704(20 \%)$ & $<0.05$ & & $<0.001$ \\
\hline
\end{tabular}


Table 2 continued

\begin{tabular}{|c|c|c|c|c|c|c|c|}
\hline \multirow{2}{*}{$\begin{array}{l}\text { Patient baseline demographics } \\
\text { and disease characteristics }\end{array}$} & \multirow{2}{*}{$\begin{array}{l}\text { Latent Class } \\
1 \\
(N=10,812)\end{array}$} & \multirow{2}{*}{$\begin{array}{l}\text { Latent } \\
\text { Class } 2 \\
(N=6728)\end{array}$} & \multirow{2}{*}{$\begin{array}{l}\text { Latent } \\
\text { Class } 3 \\
(N=2255)\end{array}$} & \multirow{2}{*}{$\begin{array}{l}\text { Latent } \\
\text { Class } 4 \\
(N=3440)\end{array}$} & \multicolumn{3}{|l|}{$p$ value $^{\mathrm{a}}$} \\
\hline & & & & & $\begin{array}{l}\text { [2] vs. } \\
{[1]}\end{array}$ & $\begin{array}{l}{[3] \text { vs. }} \\
{[1]}\end{array}$ & $\begin{array}{l}{[4] \text { vs. }} \\
{[1]}\end{array}$ \\
\hline Other antihypertensive agent ${ }^{b}$ & $2716(25 \%)$ & $1978(29 \%)$ & $360(16 \%)$ & $1108(32 \%)$ & $<0.05$ & $<0.01$ & $<0.001$ \\
\hline
\end{tabular}

Data in table are presented as the mean \pm standard deviation (SD) or as an absolute number with the percentage in parenthesis

The four latent classes are described in Table 1 and in section "Latent Class Analysis"

$T 2 D M$ Type 2 diabetes mellitus, $A C E$ angiotensin converting enzyme, $A R B$ angiotensin receptor blockers

a Latent Classes 2-4 were compared to Latent Class 1 , respectively

b Other antihypertensive agents included direct renin inhibitors, antiadrenergic antihypertensives, selective aldosterone receptor antagonists, agents for pheochromocytoma, vasodilators, monoamine oxidase inhibitors, and antihypertensive combinations

comorbidity/high treatment, moderate comorbidity/high insulin use, and high comorbidity/moderate treatment groups were 2.1, 2.0, 2.2 , and 1.7 years, respectively. The respective 1-, 3-, 5-year DN reversal rates were 7.1, 12.0, and $13.9 \%$ for the high comorbidity/moderate treatment group; $5.0,8.9$, and $8.9 \%$ for the moderate comorbidity/high insulin group; 6.1, 9.9, and $10.5 \%$ for the low comorbidity/high treatment group; and 4.5, 7.9, and 8.9\% for the low comorbidity/low treatment group (Fig. 3). The low comorbidity/high treatment and high comorbidity/moderate treatment groups were

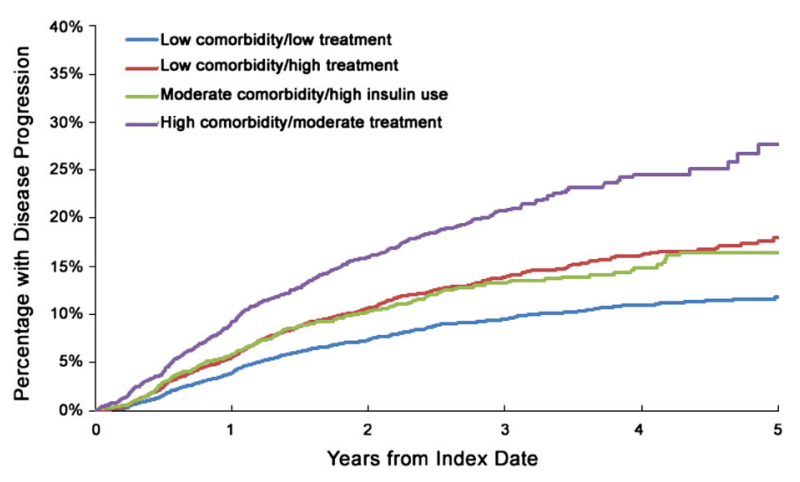

Fig. 2 Time to progression to a more severe diabetic nephropathy $(D N)$ disease stage. The respective 1-, 3-, 5-year DN progression rates were 9.27, 20.79, and 27.70\% for the high comorbidity/moderate treatment group (purple); 5.86, 13.26, and $16.46 \%$ for the moderate comorbidity/high insulin group (green); 5.60, 13.93, and $17.97 \%$ for the low comorbidity/high treatment group (red); and 3.95, 9.54, and $11.78 \%$ for the low comorbidity/ low treatment group (blue) associated with a significantly higher rate of disease reversal compared to the low comorbidity/low treatment patients (all $p<0.05$ ).

\section{All-Cause and DN-Related HRU}

Increasing comorbidity was associated with significant increases in the annual frequency of all-cause healthcare visits, with a consistent trend across inpatient, outpatient, ER, and other medical services visits $(p<0.01$ in all pairwise comparisons vs. low comorbidity/low treatment group) (Table 3). The high

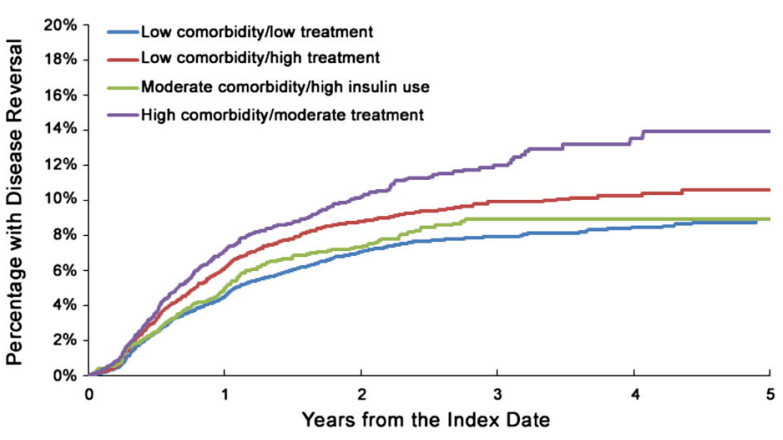

Fig. 3 Time to reversal to a less severe DN disease stage. The respective 1-, 3-, 5-year DN reversal rates were 7.09, 11.99 , and $13.92 \%$ for the high comorbidity/moderate treatment group (purple); 5.00, 8.91, and $8.91 \%$ for the moderate comorbidity/high insulin group (green); 6.11, 9.90, and $10.54 \%$ for the low comorbidity/high treatment group (red); and 4.51, 7.88, and $8.89 \%$ for the low comorbidity/low treatment group (blue) 
Table 3 All-cause and diabetic nephropathy-related healthcare resource use and healthcare costs

\begin{tabular}{|c|c|c|c|c|c|c|c|}
\hline \multirow{2}{*}{$\begin{array}{l}\text { Per patient per year HRU and } \\
\text { costs, mean } \pm S D\end{array}$} & \multirow{2}{*}{$\begin{array}{l}\text { Latent Class } 1 \\
(N=10,812)\end{array}$} & \multirow{2}{*}{$\begin{array}{l}\text { Latent Class } 2 \\
(N=6728)\end{array}$} & \multirow{2}{*}{$\begin{array}{l}\text { Latent Class } 3 \\
(N=2255)\end{array}$} & \multirow{2}{*}{$\begin{array}{l}\text { Latent Class } 4 \\
(N=3440)\end{array}$} & \multicolumn{3}{|l|}{$p$ value $^{\mathrm{a}}$} \\
\hline & & & & & $\begin{array}{l}2] \text { vs. } \\
{[1]}\end{array}$ & $\begin{array}{l}\text { [3] vs. } \\
{[1]}\end{array}$ & $\begin{array}{l}\text { [4] vs. } \\
{[1]}\end{array}$ \\
\hline \multicolumn{8}{|l|}{$\overline{\text { All-cause HRU }}$} \\
\hline Inpatient admissions & $0.09 \pm 0.29$ & $0.11 \pm 0.36$ & $0.14 \pm 0.48$ & $0.26 \pm 0.62$ & $<0.05$ & $<0.01$ & $<0.001$ \\
\hline Inpatient days & $0.44 \pm 2.23$ & $0.62 \pm 3.18$ & $0.83 \pm 3.75$ & $1.71 \pm 6.30$ & $<0.05$ & $<0.01$ & $<0.001$ \\
\hline Emergency room services & $0.39 \pm 1.12$ & $0.42 \pm 1.04$ & $0.58 \pm 2.83$ & $0.80 \pm 2.12$ & $<0.05$ & $<0.01$ & $<0.001$ \\
\hline Outpatient services & $12.96 \pm 11.26$ & $13.94 \pm 11.66$ & $14.87 \pm 13.11$ & $21.07 \pm 16.71$ & $<0.05$ & $<0.01$ & $<0.001$ \\
\hline Other $^{\mathrm{b}}$ & $1.65 \pm 3.56$ & $2.13 \pm 3.55$ & $4.22 \pm 4.93$ & $4.60 \pm 7.57$ & $<0.05$ & $<0.01$ & $<0.00$ \\
\hline \multicolumn{8}{|l|}{ DN-related HRU } \\
\hline Inpatient admissions & $0.00 \pm 0.06$ & $0.01 \pm 0.12$ & $0.02 \pm 0.17$ & $0.05 \pm 0.28$ & $<0.05$ & $<0.01$ & $<0.001$ \\
\hline Inpatient days & $0.03 \pm 0.55$ & $0.11 \pm 1.80$ & $0.15 \pm 1.47$ & $0.53 \pm 4.35$ & $<0.05$ & $<0.01$ & $<0.001$ \\
\hline Emergency room services & $0.00 \pm 0.07$ & $0.00 \pm 0.09$ & $0.01 \pm 0.15$ & $0.03 \pm 0.25$ & & $<0.01$ & $<0.001$ \\
\hline Outpatient services & $0.22 \pm 1.63$ & $0.35 \pm 1.49$ & $0.60 \pm 3.49$ & $1.46 \pm 8.17$ & $<0.05$ & $<0.01$ & $<0.001$ \\
\hline Other $^{b}$ & $0.01 \pm 0.59$ & $0.02 \pm 0.49$ & $0.05 \pm 0.63$ & $0.20 \pm 3.67$ & $<0.05$ & $<0.01$ & $<0.001$ \\
\hline \multicolumn{8}{|l|}{ All-cause healthcare costs (2016 USD) } \\
\hline Total healthcare costs & $10,172 \pm 19,518$ & $13,836 \pm 23,696$ & $17,411 \pm 25,149$ & $24,564 \pm 36,317$ & $<0.05$ & $<0.01$ & $<0.001$ \\
\hline Total medical costs & $7,383 \pm 18,161$ & $8,709 \pm 22,059$ & $11,314 \pm 23,555$ & $17,812 \pm 34,637$ & $<0.05$ & $<0.01$ & $<0.001$ \\
\hline Inpatient admission costs & $2300 \pm 11,886$ & $3107 \pm 16,511$ & $3521 \pm 14,939$ & $7616 \pm 27,065$ & $<0.05$ & $<0.01$ & $<0.001$ \\
\hline Emergency room service costs & $645 \pm 2760$ & $703 \pm 3329$ & $997 \pm 9189$ & $1581 \pm 6625$ & $<0.05$ & $<0.01$ & $<0.001$ \\
\hline Outpatient service costs & $3973 \pm 9413$ & $4252 \pm 8327$ & $4520 \pm 9125$ & $6745 \pm 10984$ & $<0.05$ & $<0.01$ & $<0.001$ \\
\hline Other medical service costs ${ }^{\mathrm{b}}$ & $466 \pm 2036$ & $646 \pm 2048$ & $2275 \pm 4455$ & $1870 \pm 5730$ & $<0.05$ & $<0.01$ & $<0.00$ \\
\hline Total pharmaceutical costs & $2788 \pm 4920$ & $5127 \pm 5828$ & $6097 \pm 6003$ & $6753 \pm 8028$ & $<0.05$ & $<0.01$ & $<0.001$ \\
\hline \multicolumn{8}{|c|}{ DN-related healthcare costs (2016 USD) } \\
\hline Total healthcare costs & $378 \pm 2088$ & $475 \pm 2088$ & $700 \pm 5015$ & $1687 \pm 11,386$ & $<0.05$ & & $<0.001$ \\
\hline Total medical costs & $113 \pm 2017$ & $166 \pm 2000$ & $474 \pm 4977$ & $1292 \pm 11,369$ & $<0.05$ & $<0.01$ & $<0.001$ \\
\hline Inpatient admission costs & $58 \pm 1500$ & $90 \pm 1589$ & $299 \pm 3800$ & $701 \pm 5867$ & $<0.05$ & $<0.01$ & $<0.001$ \\
\hline Emergency room service costs & $4 \pm 127$ & $6 \pm 137$ & $9 \pm 206$ & $28 \pm 326$ & & $<0.01$ & $<0.001$ \\
\hline Outpatient service costs & $49 \pm 1191$ & $68 \pm 744$ & $141 \pm 1746$ & $458 \pm 6318$ & $<0.05$ & $<0.01$ & $<0.001$ \\
\hline Other medical service costs ${ }^{\mathrm{b}}$ & $2 \pm 90$ & $3 \pm 84$ & $25 \pm 366$ & $105 \pm 2251$ & $<0.05$ & $<0.01$ & $<0.001$ \\
\hline Total pharmaceutical costs & $265 \pm 514$ & $309 \pm 530$ & $226 \pm 444$ & $395 \pm 624$ & $<0.05$ & $<0.01$ & $<0.001$ \\
\hline
\end{tabular}

Data in table are presented as the mean \pm SD

The four latent classes are described in Table 1

$D N$ Diabetic neuropathy, $H R U$ health resource utilization, USD U.S. dollars

${ }^{a}$ Latent Classes 2-4 were compared to Latent Class 1, respectively

b Other medical services included durable medical equipment, home care, skilled nursing facility care, and dental or vision care

comorbidity/moderate treatment group experienced a mean of 0.3 (SD 0.6) inpatient admissions annually, with a mean length of stay of 1.7 (SD 6.3) days, while the low comorbidity/ low treatment group experienced 0.1 (SD 0.3) admissions with a mean stay of 0.4 (SD 2.2) days $(p<0.001)$. For outpatient services, the high comorbidity/moderate treatment group experienced a mean of 21.1 (SD 16.7) visits annually, while the low comorbidity/low treatment group had 13.0 (SD 11.3) visits $(p<0.001)$. In addition, the high comorbidity/moderate treatment group had increased all-cause HRU compared with the low comorbidity/high treatment or moderate comorbidity/high insulin use groups in each HRU category.

A similar trend was observed regarding $\mathrm{DN}$ related HRU; patients with high comorbidity/moderate treatment had a notably high incidence of nephropathy-related inpatient, ER, 
outpatient, and other medical services visits in comparison with the other groups (all $p<0.05$ ). For example, the high comorbidity/moderate treatment group had a mean of 1.5 (SD:8.2) DNrelated outpatient visits annually, compared to 0.22 (SD 1.36) visits for the low comorbidity/ low treatment group $(p<0.001)$.

\section{All-Cause and DN-Related Healthcare Costs}

Following from the observed trend of higher HRU associated with higher comorbidity burden, higher comorbidity was also associated with significantly higher all-cause healthcare costs (Table 3). The incremental differences in total allcause healthcare costs PPPY between the low comorbidity/low treatment group and the low comorbidity/high treatment, moderate comorbidity/high insulin use, and high comorbidity/moderate treatment groups were US\$3664, US\$7239, and US\$14,392, respectively (all $p<0.05)$. Pharmaceutical cost differences (US\$2339) accounted for approximately $60 \%$ of the all-cause cost differences between the low comorbidity/high treatment and low comorbidity/low treatment groups (the next largest component was inpatient admission costs [22\%; cost difference US\$807]). Similarly, pharmaceutical cost differences (US\$3309) accounted for approximately $50 \%$ of the difference between the moderate comorbidity/high insulin use and low comorbidity/low treatment groups (the next largest components were other medical services [25\%; cost difference US\$1809] and inpatient admission [17\%; US\$1221] costs). Conversely, the majority of the cost differences between high comorbidity/moderate treatment and low comorbidity/low treatment groups was due to differences in inpatient admission costs (37\%; cost difference US\$5316), followed by pharmaceutical (28\%; US\$3965) and outpatient (19\%; US\$2772) costs.

The incremental differences in total DN-related costs PPPY between the low comorbidity/ low treatment group and the low comorbidity/ high treatment, moderate comorbidity/high insulin use, and high comorbidity/moderate treatment groups were \$US97 $(p<0.05)$, US\$322, and US\$1309 $(p<0.001)$, respectively (Table 3).
Regarding the components that were the largest contributors to total healthcare costs, a similar trend was observed in DN-related costs as with allcause costs, with pharmacy costs contributing the majority of the cost difference between the low comorbidity/low treatment and low comorbidity/high treatment groups. Higher inpatient admission costs and outpatient costs drove the increase in DN-related healthcare costs among patients with increasing comorbidity (i.e., low comorbidity/low treatment group vs. the moderate comorbidity/high insulin group and the high comorbidity/moderate treatment group).

In this study, we did not adjust for baseline characteristics in the outcome comparisons. The reason for this decision is that latent patient groups, as the main effect in the model, were identified by LCA using complications, comorbidity, and therapies for T2DM during the baseline period. Therefore, these characteristics should not be adjusted for when outcomes are compared across latent patient groups. In addition, baseline demographics are in general comparable across groups.

\section{DISCUSSION}

A large proportion of patients with diabetes develop DN, leading to progressive increases in albuminuria, declining GFR, and risk of ESRD [1]. Thus, an important step towards improving the management, treatment, and clinical outcomes of patients with T2DM is to identify patients at high risk of DN progression. To the best of our knowledge, this is the first study that has utilized LCA to identify subgroups of T2DM patients based on their diabetes comorbidity and treatment profile within a claims database, and that has compared the clinical trajectories of DN, HRU, and costs among the identified subgroups. Patients with larger burdens of comorbidity had significantly increased risk of progression to a more severe stage of DN, as well as higher all-cause and DN-related HRU and healthcare costs. In particular, patients with high comorbidity and moderate treatment use had the highest 5-year DN progression rate, HRU, and costs compared to all of the other groups. The current results demonstrate the 
feasibility of using LCA to identify clinical subgroups using many aspects of diabetes severity, and the validity of the identified subgroups was supported by the differential DN progression, resource use, and costs observed across the subgroups.

Previous research has helped establish the association between DN progression and diabetes complications or anti-diabetic treatments. Hypertension, a common complication of diabetes, plays a major role in the onset and progression of $\mathrm{DN}$, and anti-hypertensive treatment can reduce albuminuria and slow the progression of DN $[26,27]$. Remission of very advanced DN has been observed among type 1 diabetes mellitus (T1DM) and T2DM patients undergoing aggressive anti-hypertensive treatment [28-31]. In the present study, the high comorbidity/moderate treatment group had the highest prevalence of hypertension (94.7\%) and the highest rates of DN progression (5-year rate $27.7 \%$ ) among all groups. The low comorbidity/ high treatment group had the next highest prevalence of hypertension (64\%), as well as the next highest rate of $\mathrm{DN}$ progression (5-year rate $18.0 \%$ ). A previous retrospective analysis reported that the concomitant presence of retinopathy was significantly associated with DN progression in patients with T2DM [32], perhaps due to similar underlying contributing pathology. In the present study, the low comorbidity/low treatment group had a much lower prevalence of retinopathy $(2.6 \%)$ than did the other groups (range 13.1-26.8\%), as well as the lowest rate of DN progression (5-year rate $11.8 \%$ vs. [range] $16.5-27.7 \%$ ).

In addition, several studies have shown that DN progression can be slowed with intensive glycemic control [33-35]. The randomized trial ACCORD reported that therapy targeting HbA1c to levels of $<6.0$ vs. $7.0-7.9 \%$ delayed the onset of severely increased albuminuria and some microvascular complications, but increased risk mortality and other complications (e.g., hypoglycemia) [34]. A similar, prospective study reported that anti-diabetic therapy targeting HbA1c to levels of $<6.5 \%$ resulted in delayed onset of $\mathrm{DN}$, retinopathy, and neuropathy in comparison to conventional therapy [33]. These prior findings may help explain why group of patients in the current study with moderate comorbidity/high insulin use had lower rates of DN progression (5-year rate $16.5 \%$ ) than the group with low comorbidity/high treatment use (5-year rate $18.0 \%$ ).

A small number of studies have previously examined the relationship between T2DM severity and healthcare costs, reporting findings similar to the present results. A 2013 systematic literature review by Banerji et al. synthesized studies reporting the impact of glycemic control and treatment adherence, which are important contributors to DN and other diabetes complications, on the healthcare costs of T2DM patients [36]. These authors observed that the healthcare resource utilization and costs associated with T2DM management were reduced when glycemic levels and comorbidity were better controlled, although comorbid conditions were still prevalent and anti-diabetic medication adherence was largely suboptimal [37-40]. A retrospective study in 2010 by Menzin et al. reported that the hospitalization rate and healthcare costs were significantly higher for T1DM and T2DM patients with poorly controlled versus well-controlled blood glucose (>10 vs. $<7 \%$ HbA1c) [41]. Furthermore, patients with severe complications or comorbidities related to T2DM have been shown to incur healthcare costs that are threefold higher than those of matched patients without comorbidities [42], similar to the present results. Our results emphasize the magnitude of this cost difference and may present opportunities to identify patients at risk of higher healthcare expenditures.

The results of this study have potential implications for the management of T2DM and $\mathrm{DN}$ as well as for the further application of this method to identify high-risk patients based on claims data. The heterogeneous nature of T2DM complicates clinical management, and the current results indicate that some patients (e.g., the high comorbidity/moderate treatment group) may have received suboptimal anti-diabetic treatment or suboptimal blood pressure control, which led to a high risk of DN progression. However, although patients with the highest 
comorbidity were at the highest risk of $\mathrm{DN}$ progression, the 5-year rates of DN reversal among both the low comorbidity/high treatment group (10.5\%) and the high comorbidity/moderate treatment group (13.9\%) were higher than those of the low comorbidity/low treatment and moderate comorbidity/high insulin use groups (both $8.9 \%$ ). This result suggests that anti-diabetic treatments can be effective in slowing or reversing $\mathrm{DN}$ even in patients with more severe T2DM. Additionally, the LCA results highlight that there may be interactions between comorbid conditions, T2DM treatment, and patient characteristics as there are no clear indicators of group membership, i.e., group membership as a proxy for diabetes severity is not clearly defined by number or type of T2DM medications or number of co-morbidities, as would be the case in traditional claims database studies. Future research is warranted to identify any unmet treatment needs in this patient population, to characterize patients with high risk of DN progression, and to identify groups with the largest economic burden related to T2DM and DN. In addition, the current study demonstrates the potential for LCA to be used to identify classes of patients with clinically distinct T2DM profiles using information available in commercial claims data. This is an important contribution, as claims databases represent large and readily accessible sources of healthcare information but may lack data typically used to indicate prognosis (e.g., HbA1c). Future research using LCA could further contribute to understanding and reducing the risk of $\mathrm{DN}$ progression and improving clinical outcomes for patients with T2DM.

\section{Limitations}

Estimating T2DM disease severity using data from claims databases can be challenging due to the limited amount and types of clinical information they contain. Laboratory measures, such as HbA1c levels, are useful indicators of diabetes severity [43, 44], although claims databases contain limited proportions of patients with HbA1c lab values available. The
Diabetes Complication Severity Index (DCSI), a 13-point scale for scoring patients' diagnostic, pharmacy, and laboratory data, has also been used to assess healthcare costs and comorbidity management [45-47]. However, deriving the DSCI from claims data is again restrained by the availability of laboratory information in claims databases. In the present study, we derived different indicators of T2DM disease severity from the claims database, which is also subject to the limitations introduced by the data source. First, DN severity may be misclassified due to the use of the results of a single urine albumin test to define moderately increased and severely increased albuminuria. To maximize availability data to assess DN progression or reversal and because the urine albumin test data were limited, we did not require a confirmatory urine albumin test. Second, patients with T2DM were identified by ICD-9-CM codes in medical services claims; diagnoses or procedures related to ESRD, transplantation, or dialysis were also identified using diagnostic or procedural codes in the claims database. However, such diagnoses and procedures may be over- or under-reported in the data. Similarly, rates of DN progression may have been underestimated, as the data do not contain complete information regarding laboratory values. Finally, only patients with a urine albumin test were included in the study, which may not capture all patients with DN disease status since alternative laboratory tests, such as proteinuria and estimated GFR decline, may also indicate DN [48]. Although the database is geographically representative of the USA, the data include only commercially insured patients and those who have commercial insurance in supplement to their Medicare insurance. Thus, the current results may not be generalizable across different study populations that may be relevant, such as various socioeconomic groups or the uninsured.

\section{CONCLUSIONS}

This exploratory study demonstrated the feasibility of using LCA to identify patient groups with distinct clinical profiles of T2DM severity and explored the association between T2DM 
disease severity, DN progression or reversal, and economic outcomes. Increasing levels of comorbidity were generally associated with higher HRU, healthcare costs, and risk of DN progression, while anti-diabetic treatment appeared to slow DN progression.

\section{ACKNOWLEDGEMENTS}

Funding. Sponsorship for this study and article processing charges were funded by Takeda Development Center Americas, Inc. All authors had full access to all of the data in this study and take complete responsibility for the integrity of the data and accuracy of the data analysis.

Medical Writing and Editorial Assistance. Editorial assistance in the preparation of this manuscript was provided by Dr. Shelley Batts of Analysis Group, Inc. The authors would like to thank Jing Zhao from Analysis Group for significant contribution towards medical writing and analytical support. The authors would also like to thank Melvin Munsaka for his support in data analysis. This support was funded by Takeda Development Center Americans, Inc.

Authorship. All named authors meet the International Committee of Medical Journal Editors (ICMJE) criteria for authorship for this manuscript, take responsibility for the integrity of the work as a whole, and have given final approval to the version to be published.

Disclosures. Ruixuan Jiang was supported by the University of Illinois/Takeda Health Economics and Outcomes Research Fellowship. Ernest Law was also supported by the University of Illinois/Takeda Health Economics and Outcomes Research Fellowship. Zhou Zhou is an employee of Analysis Group, Inc., which has received consulting fees from Takeda Pharmaceuticals. Hongbo Yang is also an employee of Analysis Group, Inc., which has received consulting fees from Takeda Pharmaceuticals. Additionally, Eric Q. Wu is an employee of Analysis Group, Inc., which has received consulting fees from Takeda Pharmaceuticals. At the time of analysis, Raafat Seifeld was an employee of Takeda, and held Takeda stock or stock options.

Compliance with Ethics Guidelines. All procedures followed were in accordance with the ethical standards of the responsible committee on human experimentation (institutional and national) and with the Helsinki Declaration of 1964, as revised in 2013. Only de-identified data was used in this study, thus no institutional review was required. This article is based on previously conducted studies and does not contain any studies with human participants or animals performed by any of the authors.

Data Availability. The datasets generated during and/or analyzed during the current study are not publicly available due to confidentiality agreement.

Open Access. This article is distributed under the terms of the Creative Commons Attribution-NonCommercial 4.0 International License (http://creativecommons.org/licenses/ by-nc/4.0/), which permits any noncommercial use, distribution, and reproduction in any medium, provided you give appropriate credit to the original author(s) and the source, provide a link to the Creative Commons license, and indicate if changes were made.

\section{REFERENCES}

1. Tuttle KR, Bakris GL, Bilous RW, et al. Diabetic kidney disease: a report from an ADA consensus conference. Diabetes Care. 2014;37:2864-83.

2. Chen J. Diabetic nephropathy: scope of the problem. In: Lerma EV, Batuman V, eds. Diabetes and Kidney Disease. Heidelberg: Springer; 2014. p. 1-14.

3. Lim A. Diabetic nephropathy-complications and treatment. Int $\mathrm{J}$ Nephrol Renovasc Dis. 2014;7:361-81.

4. Valmadrid CT, Klein R, Moss SE, Klein BE. The risk of cardiovascular disease mortality associated with microalbuminuria and gross proteinuria in persons with older-onset diabetes mellitus. Arch Intern Med. 2000;160:1093-100. 
5. Kim JJ, Hwang BH, Choi IJ, et al. A prospective twocenter study on the associations between microalbuminuria, coronary atherosclerosis and long-term clinical outcome in asymptomatic patients with type 2 diabetes mellitus: evaluation by coronary CT angiography. Int J Cardiovasc Imaging. 2015;31:193-203.

6. Ahmad J. Management of diabetic nephropathy: recent progress and future perspective. Diabetes Metab Syndr. 2015;9:343-58.

7. de Boer IH, Rue TC, Hall YN, Heagerty PJ, Weiss NS, Himmelfarb J. Temporal trends in the prevalence of diabetic kidney disease in the United States. JAMA. 2011;305:2532-9.

8. Afkarian M, Zelnick LR, Hall YN, et al. Clinical manifestations of kidney disease among US adults with diabetes, 1988-2014. JAMA. 2016;316:602-10.

9. Haneda M, Utsunomiya $K$, Koya D, et al. A new classification of diabetic nephropathy 2014: a report from joint committee on diabetic nephropathy. J Diabetes Investig. 2015;6:242-6.

10. de Boer IH, Rue TC, Cleary PA, et al. Long-term renal outcomes of patients with type 1 diabetes mellitus and microalbuminuria: an analysis of the diabetes control and complications trial/epidemiology of diabetes interventions and complications cohort. Arch Intern Med. 2011;171:412-20.

11. Karalliedde J, Gnudi L. Diabetes mellitus, a complex and heterogeneous disease, and the role of insulin resistance as a determinant of diabetic kidney disease. Nephrol Dial Transplant. 2016;31:206-13.

12. Adler AI, Stevens RJ, Manley SE, et al. Development and progression of nephropathy in type 2 diabetes: the United Kingdom Prospective Diabetes Study (UKPDS 64). Kidney Int. 2003;63:225-32.

13. Lizicarova D, Krahulec B, Hirnerova E, Gaspar L, Celecova Z. Risk factors in diabetic nephropathy progression at present. Bratisl Lek Listy. 2014;115:517-21.

14. Duran-Perez EG, Almeda-Valdes P, Cuevas-Ramos D, Campos-Barrera E, Munoz-Hernandez L, GomezPerez FJ. Treatment of metabolic syndrome slows progression of diabetic nephropathy. Metab Syndr Relat Disord. 2011;9:483-9.

15. Araki S, Haneda M, Sugimoto T, et al. Factors associated with frequent remission of microalbuminuria in patients with type 2 diabetes. Diabetes. 2005;54:2983-7.

16. Pathak RD, Schroeder EB, Seaquist ER, et al. Severe hypoglycemia requiring medical intervention in a large cohort of adults with diabetes receiving care in U.S. integrated health care delivery systems: 2005-2011. Diabetes care. 2015;39(3):363-70.

17. Lanza ST, Rhoades BL. Latent class analysis: an alternative perspective on subgroup analysis in prevention and treatment. Prev Sci. 2013;14:157-68.

18. Virtanen M, Vahtera J, Head J, et al. Work disability among employees with diabetes: latent class analysis of risk factors in three prospective cohort studies. PLoS One. 2015;10:e0143184.

19. Fitzpatrick SL, Coughlin JW, Appel LJ, et al. Application of latent class analysis to identify behavioral patterns of response to behavioral lifestyle interventions in overweight and obese adults. Int $\mathrm{J}$ Behav Med. 2015;22:471-80.

20. Dey A, Chakraborty A, Majumdar K, Mandel A. Application of latent class analysis to estimate susceptibility to adverse health outcomes based on several risk factors. Int $\mathrm{J}$ Com Med Pub Health. 2016;3:3423-9.

21. Jiang L, Beals J, Zhang L, et al. Latent class analysis of stages of change for multiple health behaviors: results from the special diabetes program for indians diabetes prevention program. Prev Sci. 2012;13:449-61.

22. Quan H, Sundararajan V, Halfon P, et al. Coding algorithms for defining comorbidities in ICD-9-CM and ICD-10 administrative data. Med Care. 2005;43:1130-9.

23. Gross JL, de Azevedo MJ, Silveiro SP, Canani LH, Caramori ML, Zelmanovitz T. Diabetic nephropathy: diagnosis, prevention, and treatment. Diabetes Care. 2005;28:164-76.

24. Bureau of Labor Statistics (U.S. Department of Labor). Consumer price index 2016 [Jan 1, 2017]. Available from: https://www.bls.gov/cpi/.

25. Lanza ST, Collins LM, Lemmon DR, Schafer JL. PROC LCA: a SAS procedure for latent class analysis. Struct Equ Model. 2007;14:671-94.

26. Rossing K. Progression and remission of nephropathy in type 2 diabetes: new strategies of treatment and monitoring. Dan Med Bull. 2007;54:79-98.

27. Parving $\mathrm{HH}$, Hovind $\mathrm{P}$, Rossing $\mathrm{K}$, Andersen $\mathrm{S}$. Evolving strategies for renoprotection: diabetic nephropathy. Curr Opin Nephrol Hypertens. 2001;10:515-22.

28. Hebert LA, Bain RP, Verme D, et al. Remission of nephrotic range proteinuria in type I diabetes. Collaborative study group. Kidney Int. 1994;46:1688-93. 
29. Hovind P, Rossing P, Tarnow L, Toft H, Parving J, Parving $\mathrm{HH}$. Remission of nephrotic-range albuminuria in type 1 diabetic patients. Diabetes Care. 2001;24:1972-7.

30. Hovind P, Tarnow L, Rossing P, Carstensen B, Parving HH. Improved survival in patients obtaining remission of nephrotic range albuminuria in diabetic nephropathy. Kidney Int. 2004;66:1180-6.

31. Rossing K, Christensen PK, Hovind P, Parving HH. Remission of nephrotic-range albuminuria reduces risk of end-stage renal disease and improves survival in type 2 diabetic patients. Diabetologia. 2005;48:2241-7.

32. Alwakeel JS, Isnani AC, Alsuwaida A, et al. Factors affecting the progression of diabetic nephropathy and its complications: a single-center experience in Saudi Arabia. Ann Saudi Med. 2011;31:236-42.

33. Ohkubo Y, Kishikawa H, Araki E, et al. Intensive insulin therapy prevents the progression of diabetic microvascular complications in Japanese patients with non-insulin-dependent diabetes mellitus: a randomized prospective 6-year study. Diabetes Res Clin Pract. 1995;28:103-17.

34. Ismail-Beigi F, Craven T, Banerji MA, et al. Effect of intensive treatment of hyperglycaemia on microvascular outcomes in type 2 diabetes: an analysis of the ACCORD randomised trial. Lancet. 2010;376:419-30.

35. Callaghan BC, Little AA, Feldman EL, Hughes RA. Enhanced glucose control for preventing and treating diabetic neuropathy. Cochrane Database Syst Rev. 2012;6:CD007543.

36. Banerji MA, Dunn JD. Impact of glycemic control on healthcare resource utilization and costs of type 2 diabetes: current and future pharmacologic approaches to improving outcomes. Am Health Drug Benefits. 2013;6:382-92.

37. Stark Casagrande S, Fradkin JE, Saydah SH, Rust KF, Cowie CC. The prevalence of meeting A1C, blood pressure, and LDL goals among people with diabetes, 1988-2010. Diabetes Care. 2013;36:2271-9.

38. Cramer JA. A systematic review of adherence with medications for diabetes. Diabetes Care. 2004;27:1218-24.
39. Ho PM, Rumsfeld JS, Masoudi FA, McClure DL, Plomondon ME, Steiner JF, et al. Effect of medication nonadherence on hospitalization and mortality among patients with diabetes mellitus. Arch Intern Med. 2006;166:1836-41.

40. Wild $\mathrm{H}$. The economic rationale for adherence in the treatment of type 2 diabetes mellitus. Am J Manag Care. 2012;18:S43-8.

41. Menzin J, Korn JR, Cohen J, et al. Relationship between glycemic control and diabetes-related hospital costs in patients with type 1 or type 2 diabetes mellitus. JMCP. 2010;16:264-75.

42. Gandra SR, Lawrence LW, Parasuraman BM, Darin RM, Sherman JJ, Wall JL. Total and component health care costs in a non-Medicare HMO population of patients with and without type 2 diabetes and with and without macrovascular disease. JMCP. 2006;12:546-54.

43. Lipska KJ, Warton EM, Huang ES, et al. HbA1c and risk of severe hypoglycemia in type 2 diabetes: the diabetes and aging study. Diabetes Care. 2013;36:3535-42.

44. Zhao W, Katzmarzyk PT, Horswell R, Wang Y, Johnson J, Hu G. HbA1c and coronary heart disease risk among diabetic patients. Diabetes Care. 2014;37:428-35.

45. Selby JV, Karter AJ, Ackerson LM, Ferrara A, Liu J. Developing a prediction rule from automated clinical databases to identify high-risk patients in a large population with diabetes. Diabetes Care. 2001;24:1547-55.

46. Rosenzweig JL, Weinger K, Poirier-Solomon L, Rushton M. Use of a disease severity index for evaluation of healthcare costs and management of comorbidities of patients with diabetes mellitus. Am J Manag Care. 2002;8:950-8.

47. Young BA, Lin E, Von Korff M, et al. Diabetes complications severity index and risk of mortality, hospitalization, and healthcare utilization. Am J Manag Care. 2008;14:15-23.

48. Porrini E, Ruggenenti P, Mogensen CE, et al. Nonproteinuric pathways in loss of renal function in patients with type 2 diabetes. Lancet Diabetes Endocrinol. 2015;3:382-91. 